GISELA HARRAS

\title{
Eine Möglichkeit der kontrastiven Analyse von Kommunikationsverben
}

Zusammenfassung: In dem folgenden Beitrag wird eine sprachunabhängige Basis entwickelt zur Beschreibung von Sprechaktverben fur jede beliebige Sprache. Die Tragfähigkeit dieser Basis wird an zwei Beispielen verdeutlicht: einmal an der Demonstration von Lexikalisierungstendenzen fur das Deutsche und zum andern an der Kontrastierung der Strukuren des deutschen und des englischen Wortfelds für "lügen".

Summary: The following contribution will deal with the possitility of constituting a conceptual base for describing speech act verbs indepently of any language. The utility of this base will be shown by two examples: first by the demonstration of some cases of lexicalization in German and secondly by contrasting the structures of the German and the English wordfield for "to lie".

Résume: La contribution suivante contient une conception d'une base universelle pour décrire des verbes de communication d'une langue quelconque. La capacité de cette base sera élucidé en mettant en relief quelque cas de lexicalisations en allemand et en contrastant la structure du champ de mot allemand pour "mentir" avec celle du champ de mot anglais.

\section{Eine Basis für Kontraste: Rekurssituationstypen}

Alle Sprachen verfügen uber Ausdrucke, mit denen auf sprachliche Handlungen Bezug genommen werden kann, d.h. auf Situationen, die ganz allgemein dadurch charakterisiert sind, daß jemand, ein Sprecher, einem Hörer im Sinn von angesprochenem Adressaten, etwas mit einer bestimmten Absicht sagt. Dieser Typ von Situation mit seinen standardmäßigen Situationsrollen - einem Sprecher, einem Hörer, einem Äußerungprodukt sowie einer komplexen kommunikativen Einstellung des Sprechers - wird als ein konzeptuelles Setting verstanden, das konstitutiv ist für die Verwendungsmöglichkeiten der entsprechenden Prädikate in einer beliebigen Sprache. Ich nenne ihn den zugrundeliegenden allgemeinen Rekurssituationstyp, der die Basis liefert fur weitere Spezifikationen, indem den entsprechenden Situationsrollen bestimmte Eigenschaften (Attribute) zugeordnet werden, für die jeweils bestimmte Ausprägungen dieser Eigenschaften (Attributwerte) spezifiziert werden können. Die relevanten Eigenschaften sind die folgenden:

(1) für das Äußerungsprodukt die Eigenschaft seines propositionalen Gehalts;

(2) für die kommunikative Einstellung des Sprechers die Eigenschaft seiner Einstellung zu dem Gesagten, z.B. ob er es glaubt, will oder gut bzw. schlecht findet, technisch ge- 
sprochen: die propositionale Einstellung sowie die Eigenschaft der intentionalen Einstellung oder Sprecherabsicht und weitere situative Bedingungen aus der Sicht des Sprechers, die zusammenfassend Interaktionswelt des Sprechers genannt werden soll.

Durch die Zuordnung dieser Eigenschaften zu den genannten Rollen sowie die weitere Zuordnung der Ausprägung dieser Eigenschaften werden spezielle Rekurssituationstypen aufgebaut, die jeweils den konzeptuellen Rahmen abgeben für die Klassifizierung von Teilmengen kommunikativer Verben.

Für die Eigenschaften des propositionalen Gehalts gibt es die folgenden Optionen:

Jeder propositionale Gehalt repräsentiert einen Geschehenstyp, der als Zustand, Ereignis oder Handlung ausgeprägt bzw. diesbezüglich auch unbestimmt sein kann. Im Fall, daß der Geschehenstyp eine Handlung darstellt, gibt es verschiedene Ausprägungen des Rollenbezugs, je nachdem ob der Handlungsträger der Sprecher (z.B. bei versprechen), der Hörer (z.B. bei bitten) oder Sprecher \& Hörer (z.B. bei vorschlagen) ist.

Für alle Ausprägungen des Geschehenstyps gibt es Optionen für ihren Zeitbezug: vergangen (z.B. fur tadeln), gegenwärtig (?), zuküftig (z.B. für ankündigen) sowie die Option der Unbestimmtheit des Zeitbezugs (z.B. fur informieren).

Insgesamt ergibt sich somit die folgende Optionsverteilung für die Ausprägungen des propositionalen Gehalts:

Figur 1.

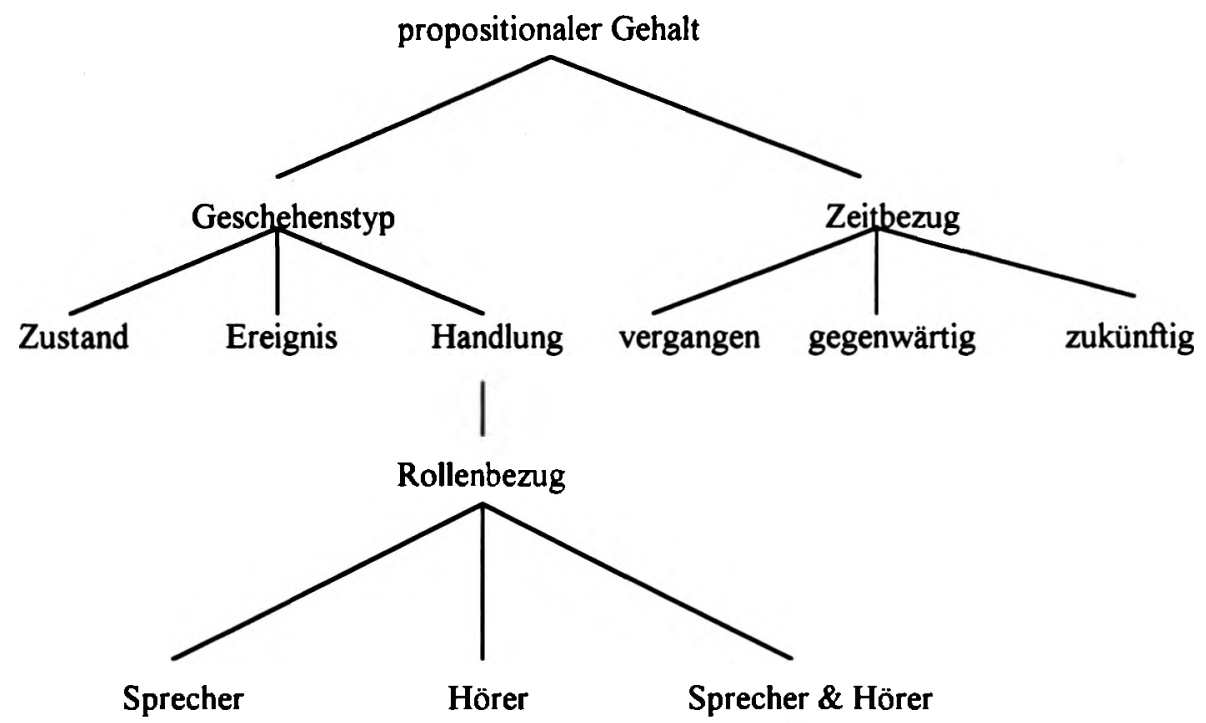

Für die propositionale Einstellung des Sprechers werden fünf Möglichkeiten von Eigenschaf tsausprägungen vorgesehen:

- epistemische Einstellung

- voluntative Einstellung

- ordinative (einstufende) Einstellung 
- evaluative Einstellung

- emotive Einstellung

Epistemische Einstellungen werden durch die Prädikate für wahr halten und kennen gekennzeichnet (zur Diskussion einer kommunikativen Epistemik vgl. Mudersbach (1984)). Im einzelnen ergibt dies die folgenden Verteilungen:
$S$ hält fur wahr: $P$
(z.B. behaupten)
$S$ hält fur wahr: nicht $P$
(z.B. bestreiten)
$S$ hält nicht für wahr: $P$
(z.B. lilgen)
$S$ hält nicht fur wahr: nicht $P$,
(?)
$S$ hält für wahr: $P^{\prime}$ ist zu $P$ ergänzbar
(z.B. fragen)
$S$ kennt: $P$
(z.B. mitteilen)
S kennt nicht: $P$
(z.B. fragen)

Voluntative Einstellungen sind durch das Prädikat wollen gekennzeichnet (zur Semantik vgl. v. Wright (1977)). Es ergeben sich die folgenden Verteilungen:
S will: $P$
(z.B. bitten)
$S$ will: nicht $P$
(z.B. verbieten)
S will nicht: $P$
$S$ will nicht: nicht $P$
(z.B. warnen)
(?)

Die Differenzierung des Negationsskopus in der Einstellung einerseits und im Einstellungs halt andererseits ist durch das Kriterium der Thematisierung von $P$ im Vorkontext (explizit oder implizit) begrundet, z.B. keine Thematisierung von $\mathrm{P}$ für lügen, warnen vs. Thematisierung von $P$ fur bestreiten, verbieten.

Ordinative (einstufende Einstellungen) sind durch das Prädikat finden gekennzeichnet (zu Einstufungen vgl. Urmson (1974)):
$S$ findet: $P$
(z.B. beurteilen)
$S$ findet: nicht $P$
(z.B. ablehnen)

Evaluative Einstellungen sind durch das Prädikat gut/schlecht finden gekennzeichnet:
$S$ findet: $P$ gut
(z.B. rühmen)
S findet: $P$ schlecht
(z.B. kritisieren)

Emotive Einstellungen sind durch das Prädikat empfinden gekennzeichnet:

S empfindet Freude (Ärger, Leid) wegen P

(z.B. jubilieren, lamentieren)

Intentionale Einstellungen (Sprecherabsichten) sind durch das Prädikat wollen (vgl. v. Wright (19T7)) gekennzeichnet:

$S$ will: $H$ tut $P$

$S$ will: $H$ tut nicht $P$

$S$ will: $H$ tut $R$

$S$ will: $H$ hält fur wahr: $P$

$S$ will: $H$ hält fur wahr: nicht $P$

$S$ will: $H$ hält nicht fur wahr. $P$

$S$ will: $H$ hält nicht für wahr. nicht $P$
(z.B. anordnen)

(z.B. verbieten)

(z.B. fragen)

(z.B. bekräftigen)

(z.B. bestreiten)

(z.B. berichtigen)

(?) 
S will: $H$ kennt $P$

$S$ will: $H$ kennt nicht: $P$

S will: $\mathrm{H}$ findet: $\mathrm{P}$

$S$ will: $H$ findet: nicht $P$

$S$ will: $H$ findet: $P$ gut/schlecht

S will: $Q$

(Q steht fur eine institutionelle Tatsache)

$S$ will: $H$ erkennt: $\Phi(S)$
(z.B. mitteilen)

(z.B. verheimlichen)

(z.B. plädieren für)

(z.B. ausreden)

(z.B. loben, tadeln)

(z.B. taufen)

(z.B. versprechen, wehklagen)

( $\Phi$ steht für eine propositionale oder intentionale Einstellung)

Fur die Interaktionswelt aus der Sicht des Sprechers gibt es neben kulturspezifischen Ausprägungen wie die Gebundenheit an bestimmte Institutionen die folgenden systematischen Ausprägungen:

- die Planzierung der Äußerung: initial (z.B. fragen), reaktiv (z.B. bestreiten), re-reaktiv (2.B. beharren auf)

- bestimmte Vorannahmen des Sprechers bezuglich relevanter Situationsumstände:

erwartbar: $P$

erwartbar, nicht $P$

nicht erwartbar: $P$

nicht erwartbar: nicht $P$

im Interesse von S: $\mathbf{P}$

im Interesse von $S$ : nicht $P$

nicht im Interesse von S: $P$

nicht im Interesse von $S$ : nicht $P$

im Interesse von H: P

im Interesse von $\mathrm{H}$ : nicht $P$

nicht im Interesse von $\mathrm{H}$ : $\mathrm{P}$

nicht im Interesse von $\mathrm{H}$ : nicht $\mathrm{P}$

$H$ hält für wahr. $P$

$H$ hält fur wahr. nicht $P$

$H$ hält nicht fur wahr. $P$

$H$ hält nicht fur wahr. nicht $P$

H kennt: $P$

H kennt nicht: $P$

H findet: $P$ gut/schlecht

H empfindet Freude (Ärger, Trauer) wegen $P$

$H$ ist fähig, $P$ zu tun

$H$ ist fähig, $R$ zu tun
(z.B. warnen)

(z.B. ermahnen)

(z.B. raten)

(?)

(z.B. bitten)

(Z.B. verbieten)

(?)

(?)

(z.B. zusichern)

(?)

(z.B. warnen)

(?)

(z.B. zurilckweisen)

(2.B. insistieren)

(z.B. behaupten)

(?)

(z.B. fragen)

(z.B. informieren)

(z.B. schmeicheln, beleidigen)

(z.B. trösten)

(z.B. ermuntern)

(z.B. fragen) 


\section{Wozu die Basis taugt: Lexikalisierungstendenzen im Deutschen und Kon- traste von Wortfeldstrukturen Deutsch-Englisch}

Die dargestellte konzeptuelle Basis ist aus zwei Gründen so exhaustiv wie möglich angelegt:

(1) Sie soll es gestatten, den spezifisch deutschen Lexikalisierungsraum im Bereich der kommunikativen Prädikate zu erfassen. Unter Lexikalisierungsraum ist zunächst die Menge aller lexikalischen Ausdrucke zu verstehen, fur deren Bedeutung eine der möglichen Kombinationen von Attributwerten konstitutiv ist. Zum anderen soll der Begriff 'Lexikalisierungsraum' auch als Möglichkeit der Lexikalisierung von logisch möglichen Kombinationen von Attributwerten verstanden werden, d.h. er umfaßt außer den konkreten lexikalischen Einheiten des Deutschen auch mögliche Wörter (vgl. Baumgärtner (1977)). Bezuglich der Tauglichkeit der Basis furr kontrastive Untersuchungen bedeutet dies, daß zunächst einmal alle Möglichkeiten ausgeschöpft werden, um die systematischen und idiosynkratischen Spezifika einer Sprache, in dem Fall des Deutschen, zu erfassen, um dann in einem nächsten Schritt Kontraste zu einer zweiten Sprache auf dem Hintergrund der Befunde von Lexikalisierungstendenzen im Deutschen zu ermitteln. Beispiele fur Lexikalisierungstendenzen werden im folgenden Abschnitt gegeben und diskutiert werden.

(2) Die konzeptuelle Basis soll daruber hinaus - ganz unabhängig von der Lexikalisierung in einer bestimmten Sprache - eine differenzierte - wenn man so will: im traditionellen Sinn onomasiologische - Grundlage fur kontrastive lexikologische Untersuchungen abgeben, speziell fur die Organisation von lexikalischen Paradigmen oder Wortfeldern. Im letzten Abschnitt dieses Beitrags werde ich dazu ein Beispiel diskutieren.

\subsection{Lexikalislerungstendenzen im Deutschen}

Lexikalisierungstendenzen im Deutschen lassen sich nach dem Ausmaß bestimmen, in dem bestimmte kommunikative Situationstypen als spezielle Rekurssituationstypen lexikalisiert sind oder auch nicht. Eine solche Bestimmung kann und soll naturlich kein Selbstzweck bleiben: ihre Ergebnisse können Ankerpunkte liefern für anschließende kontrastive Untersuchungen. Dies gilt in besonderem Maß für diejenigen Fälle, in denen logisch mögliche Kombinationen von Attributwerten mögliche spezielle Rekurssituationstypen darstellen, für deren Bezeichnung es im Deutschen keine lexikalischen Ausdrlicke gibt. Ob diese Fälle dann auch tatsächlich Ankerpunkte fur kontrastive Untersuchungen abgeben können, hängt zusätzlich davon $a b$, welche Erklärung für eine jeweilige lexikalische Lucke gefunden werden kann.

Im folgenden werden vier Fälle von lexikalischen Lücken vorgestellt und Ansätze zu ihrer Erklärung vorgeschlagen:

(1) Die Unterscheidung der beiden möglichen Attributwerte 'Zustand' und 'Ereignis' für den Parameter 'Geschehenstyp propositionaler Gehalte' ist für das Deutsche nicht distinktiv: es gibt keine Verben speziell für die Lexikalisierung von so etwas wie "Ereignissagen" vs. "Zustandssagen"; dagegen gibt es ein "Handlungssagen", lexikalisiert durch alle direktiven und kommissiven Prädikate wie bitten, sich verpflichten, vorschlagen, raten usw. Wirklich distinktiv ist fur das Deutsche nur die Unterscheidung "Handlung" vs. "beliebiger Sachverhalt". 
Der Grund für eine solche Lexikalisierungslücke könnte in Relevanzprinzipien zu finden sein, die in einer Gruppe gelten: für eine Gruppe ist es fur ihr Überleben als Gruppe wichtig, sich uber zukunftige und vergangene Handlungen ihrer Mitglieder zu verständigen d.h. ihr Verhal ten zu koordinieren und einschlägige Konventionen auszubilden. Man sollte meinen, daß dies fur jede Gruppe gilt, so daß die Suche nach einer Sprache mit entsprechenden Lexikalisierungen vergeblich sein dürfte, aber vielleicht ist dies auch viel zu eurozentrisch gedacht!

(2) Für die Attributwerte des Parameters 'intentionale Sprechereinstellung' gibt es die beiden prinzipiellen Optionen:

$S$ will: $H$ tut $P$

$S$ will: $H$ tut $P$ nicht

Im Deutschen gibt es keine eigene Lexikalisierung fur Nicht-Tun oder Unterlassen von Hörerhandlungen bei einfachen Aufforderungen, d.h. es gibt keinen lexikalischen Ausdruck für 'auffordern, etwas nicht zu tun'. Der Einwand, das Deutsche habe das Verb verbieten ist nicht stichhaltig; verbieten heißt zwar 'auffordern, etwas nicht zu tun', impliziert aber daruber hinaus eine Art Vorkommunikation, die in etwa so formuliert werden kann: ' $\mathrm{H}$ hat den Wunsch, die Absicht geäußert, $\mathrm{P}$ zu tun'.

Das gleiche gilt für kommissive Prädikate mit sprecherrollenbezogener Handlung wie versprechen: fur 'versprechen, daß nicht P' gibt es keinen lexikalischen Ausdruck. Die Verben entsagen und verzichten implizieren ebenso wie verbieten eine Art Vorkommunikation.

Die gleiche Restriktion finden wir nun auch für repräsentative Prädikate wie behaupten, mitteilen, ankündigen usw. Für deren komplexe Gegenstucke mit implizierter Vorkommuni kation wie zustimmen, zurückweisen, bestätigen, bestreiten usw. ist die Opposition P vs. nicht $P$ distinktiv.

Eine Erklärung dieser Lexikalisierungslücke scheint auf der Hand zu liegen: die komplexen Prädikate bezeichnen Rekussituationstypen, in denen $P$ bereits durch Vorkommunikation eingefuhrt ist, d.h. P ist im Aufmerksamkeitsbereich oder in der kognitiven Umgebung von $\mathrm{S}$ und $\mathrm{H}$ manifest, so daß eine Festlegung bezuglich 'P:ja' oder 'P:nein' einen hohen Relevanzwert erhält. Diese Begrundung ist pragmatischer Natur. Ob zur Erklärung solcher Lexikalisierungslucken auch allgemeine Prinzipien zur Strukturierung formaler Repräsentationen herangezogen werden können, wäre durch eine systematische Analyse zu prüfen. Würde dann die Antwort positiv ausfallen, wäre es mußig, nach Sprachen zu suchen, in denen entsprechende lexikalische Ausdruicke existierten.

(3) Für die möglichen Attributwerte des Parameters 'intentionale Sprechereinstellung' gibt es im Deutschen keine Lexikalisierungen von Kombinationen aus ihnen. Das mag trivial erscheinen, doch denkbar wäre z.B. die folgende Kombination:

S will: $H$ hält für wahr: $P$

$S$ will: $H$ findet: $P$ gut/schlecht

Die Kombination dieser beiden Attributwerte würde z.B. Prädikate hervorbringen wie *gutneinen, *miesjahen, *schlechtneinen u.ä., Prädikate also, mit denen sowohl das Bestehen eines Sachverhalts behauptet oder supponiert als auch dieser Sachverhalt bewertet wird. Solche Prädikate gibt es im Deutschen nicht. Existente Verben wie kritisieren, gutheißen, tadeln, loben usw. sind faktive Verben, mit denen das Bestehen der jeweiligen bewerteten Sachverhalte vorausgesetzt, präsupponiert wird.

Eine vernunftige Erklärung fur diese Lexkalisierungslucke ist nicht leicht zu finden: die Kombination ist logisch möglich und ihre Lexikalisierung wurde dem Prinzip der lexika- 
lischen Ökonomie entgegenkommen. Jedoch sollte man vorsichtig sein, aus dieser Beobachtung zu schließen, daB hier ein besonders geeigneter Ankerpunkt fur kontrastive Untersuchungen vorläge: alle mir bekannten indogermanischen Sprachen verhalten sich bezuglich der Lexikalisierung der beiden Attributwerte genau wie das Deutsche.

(4) Bestimmte Attributwerte für den Parameter 'Interaktionswelt aus der Sicht des Sprechers', speziell solche fur Sprecher- und Hörerinteressen, sind für Direktive und Kommissive im Deutschen uberhaupt nicht lexikalisierbar: fur Direktive wie auffordern gibt es keine Kombination der Sprecherintention: ' $\mathrm{S}$ will: $\mathrm{H}$ tut $\mathrm{P}$ ' mit der Vorannahme: 'nicht im Interesse von H: P'. Das Prádikat zumuten, das einer solchen Kombination noch am ehesten entspräche, ist weder performativ verwendbar - allenfalls in solchen Formeln wie leider muß ich dir zumuten, daß... - noch eindeutig auf sprachliche Handlungen beziehbar. Fur Kommissive wie versprechen gilt das gleiche: fur den Fall, daß jemand jemandem etwas fur ihn schlechtes verspricht, haben wir das Verb drohen, dessen problematische Werteverteilung in Wendungen wie ich drohe dir nur ungern zum A usdruck kommt.

Schließlich sind Kombinationen mit negativen Sprecherinteressen nicht lexikalisiert: fur nicht-sprachliches, altruistisches, sich selbst nicht schonendes Handeln gibt es das Verb sich aufopfern; es gibt aber kein kommissives Prädikat, in dem sowohl die positive Wertung der Sprecherhandlung für den Hörer als auch die negative Wertung der Handlung fü den Sprecher selbst lexikalisiert wäre.

$\mathrm{DaB}$ solche Lexikalisierungen nicht vorkommen, könnte kommunikationsethische Grunde haben: mit ihnen würde eine Verletzung der angemessenen Distanz zwischen Sprecher und Hörer zum Ausdruck kommen, wobei die Angemessenheit der Distanz in der Balance zwischen der Vermeidung des Eingreifens in die Sphäre des anderen einerseits und der Vermeidung des Aufdringens der eigenen Sphäre andererseits bestünde. Allerdings ist für diese Fälle sicher in Rechnung zu stellen, daß die Variable der Angemessenheit bzw. Distanz zwischen Sprecher und Hörer in den verschiedenen Kommunikationsgemeinschaften unterschiedlich gedeutet werden kann, so daß hier in der Tat ein interessanter Ankerpunkt für kontrastive Untersuchungen - in erster Linie naturlich fur nicht-indogermanische Sprachen -vorliegen könnte.

Die hier diskutierten vier Fälle lexikalischer Lücken stellen natülich nur einen kleinen Ausschnitt dar aus dem Möglichkeitsspektrum, das die konzeptuelle Basis zur Verfugung stellt. Inwieweit die Erklärungsansätze der Lexikalisierungstendenzen im Deutschen tragen, kann nur durch eine systematische Untersuchung aller Kombinationsmöglichkeiten geklärt werden.

\subsection{Kontraste von Wortfeldstrukturen Deutsch-Englisch: das Lügen-Paradigma}

Ausgangspunkt fur die Konstituierung des Paradigmas ist der (komplexe) Aspekt des speziel len Rekurssituationstyps Repr.lug. mit den folgenden Attributwerten:

(1) propositionaler Gehalt: unbestimmt

(2) propositionale Einstellung von $S: S$ hält nicht fur wahr. $P$

(3) intentionale Einstellung von $S: S$ will: $H$ hält fur wahr. $P$

(4) Vorannahme von S: H kennt nicht: P 
Die gewăhlte Bezeichnung dieses speziellen Rekurssituationstyps trägt durch das Ettikett "Repr." der Tatsache Rechnung, daß lügen zur Klasse der Repräsentative, d.h. der Behauptens- und Mitteilungsprädikate, gehört. Relativ zu diesem speziellen Rekurssituationstyp ergeben sich fur das Deutsche die folgenden Verben:

die einfachen Verben: lilgen, flunkern, schwindeln

die präfigierten Verben: anlügen, anflunkern, anschwindeln

belïgen, beflunkern, beschwindeln

erlügen, erflunkern, erschwindeln

vorlïgen, vorflunkern, vorschwindeln

rumlügen, rumflunkern, rumschwindeln

die hybriden Ausdrucke: irrefïhren, irreleiten, vormachen

Unter hybriden Ausdrucken werden solche Prädikate verstanden, die auch, aber nicht aus schließlich sprachliche Handlungen bezeichnen (vgl. Searle/Vanderveken (1985)).

Fur das Englische ergibt sich relativ zu dem speziellen Rekurssituationstyp Repr.lüg. ein vergleichsweise armselig bestlucktes Paradigma, zu dem nur die Ausdricke to lie, to fib und allenfalls der hybride Ausdruck to mislead gehören, wobei hier komplexe Ausdrucksmöglichkeiten wie to tell lies/fibs nicht berucksichtigt werden, was auch fur das deutsche Paradigma gilt.

In beiden Pardigmen ist jeweils ein Ausdruck - im Deutschen lugen, im Englischen to lie Hyperonym. Relativ zur semantischen Information, die durch den Rekurssituationstyp Repr.lüg. repräsentiert ist, sind alle anderen Ausdrucke kohyponym zueinander.

Eine weitere Strukturierung des Lijgen-Paradigmas ergibt sich durch die Berlicksichtigung der beiden folgenden Aspekte:

(1) des Intensitätsgrades der Gewichtung der Lüge; dieser Aspekt gestattet es, flunkern und schwindeln von lilgen und irreführen/irreleiten zu unterscheiden, wobei flunkern den geringsten Intensitätsgrad besitzt und irrefilhren/irreleiten den höchsten. Das gleiche gilt furr die Unterscheidung von to fib und to lie und to mislead. Damit korreliert zugleich auch ein Unterschied im Register der Ausdrücke: flunkern, schwindeln, to fib gehören eher zur familiären Umgangssprache, lügen und to lie zur Schriftsprache;

(2) der unterschiedlichen Perspektiviertheit der Rollen des allgemeinen zugrundeliegenden Rekurssituationstyps S, H und P. Mit Perspektiviertheit der Rollen ist die jeweilige obligatorische syntaktische Realisierung als Verbkomplemente gemeint (vgl. Storrer (1991)). Für das Deutsche ergibt sich unter all den genannten Aspekten die folgende Wortfeldstruktur (Figur 2): 
Figur 2.

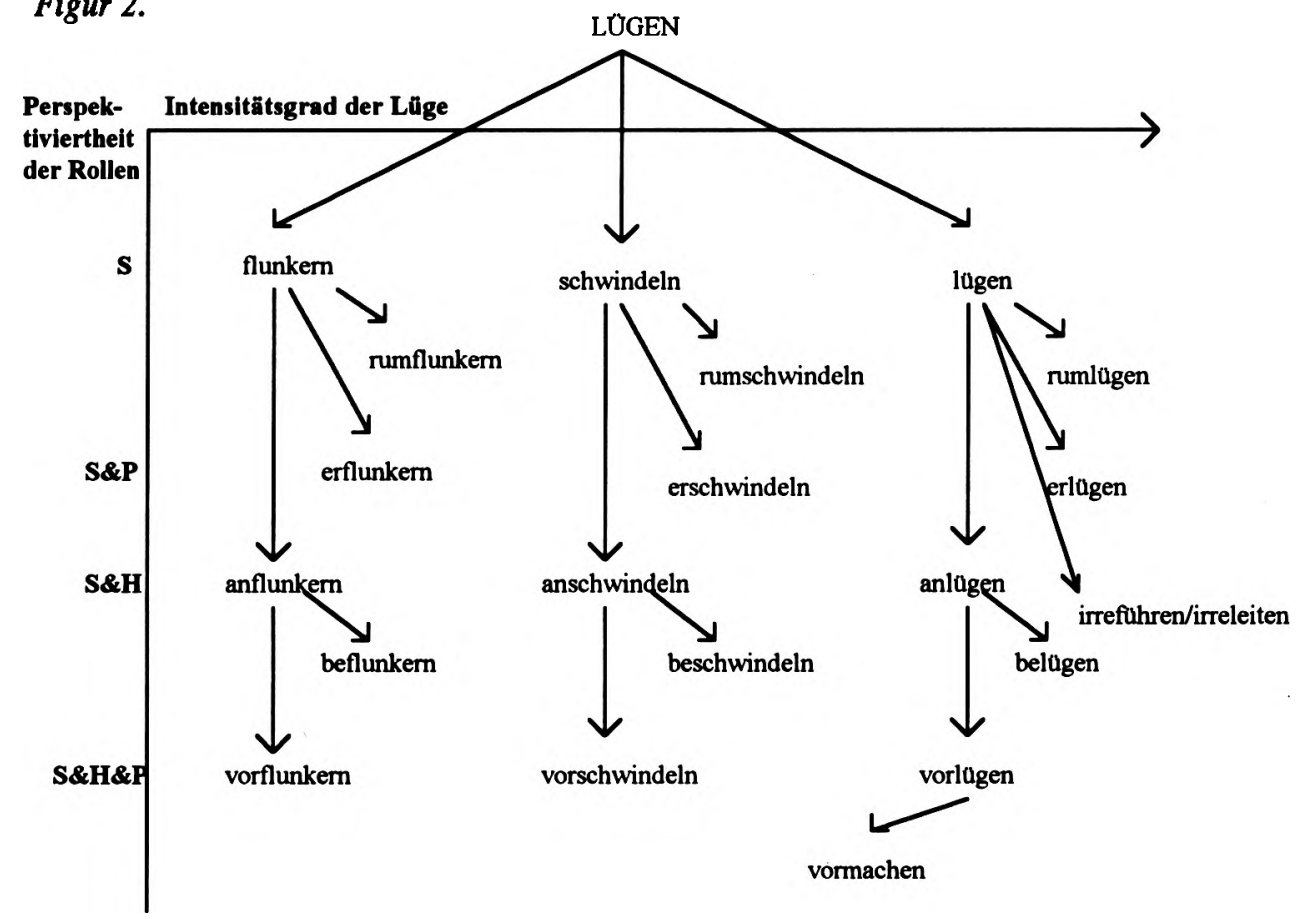

Für das Englische ergibt sich dagegen eine sehr armselige Wortfeldstruktur, die hier aus Gründen der Kontrastwirkung zusätzlich um die fakultativen syntaktischen Realisierungen für to lie ergänzt sein soll:

Figur 3.

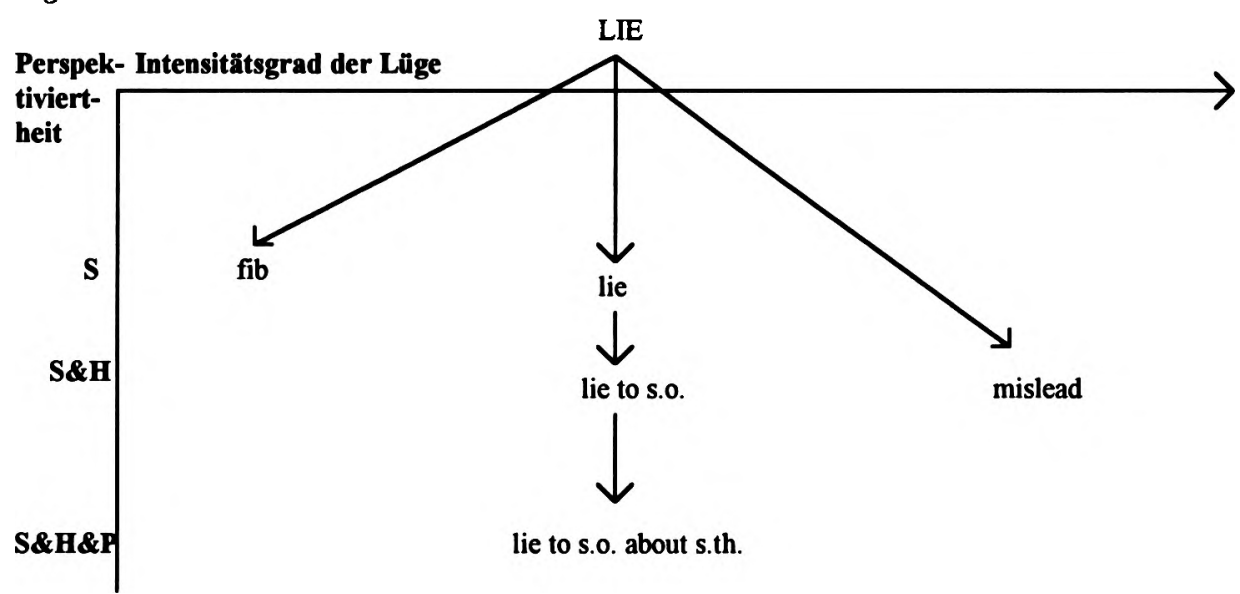

Die im Vergleich zum Englischen reichhaltigere Wortfeldstruktur des Deutschen soll im folgenden noch weiter differenziert werden: zunächst sind zwei spezielle Restriktionen festzuhalten: 
(1) rumlugen, rumflunkern, rumschwindeln blockieren die syntaktische Realisierung aller Situationsrollen außer S, vgl.:

(i) Er hat ihm gegenuber gelogen

(ii) *Er hat ihm gegenuber rumgelogen

(2) erlitgen, erflunkern, erschwindeln können nicht finit gebraucht werden, vgl.:

(iii) *Er erlog diese Geschichte

(iv) Er hat diese Geschichte erlogen

In der oben repräsentierten Wortfeldstruktur sind die be-Präfigierungen von lïgen, flunkern, schwindeln als Hyponyme zu den entsprechenden an-Präfigierungen dargestellt. Wie läßt sich diese Relation rechtfertigen? Zudem scheint die be-Präfigierung bei flunkern ungewöhnlich zu sein; jedenfalls kommt sie weder in den umfänglichen IDS-Korpora vor noch ist sie in irgendeinem deutschen Wörterbuch als Stichwort verzeichnet. Es bleibt also die Frage, ob es einen semantischen Unterschied zwischen den an- und be-Präfigierungen gibt und wie er beschrieben werden kann und wie sich die Blockierung der be-Präfigierung bei flunkern erklären läßt.

Mithilfe des Modells des allgemeinen Rekurssituationstyps und mithilfe des Kriteriums der Rollenperspektiviertheit lassen sich die beiden Probleme offenbar nicht lösen. Ich schlage deshalb vor, das Modell des allgemeinen Rekurssituationstyps, der ja zunächst nur für Kommunikationssituationen einschlägig ist, in ein generelles Ereignismodell, wie es Lutzeier vorgestellt hat, zu integrieren. Das generelle Ereignismodell enthält - lokalistisch metaphorisch gesprochen - eine source-Relation, ein goal-Relation sowie eine signpost-Relation:

Figur 4. (Lutzeier 1991:205)

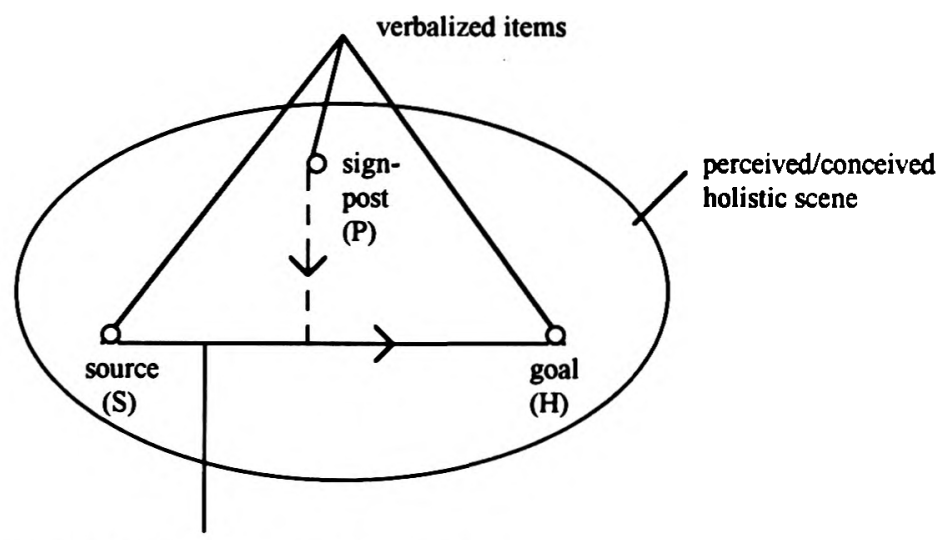

perceived/conceived (dynamic) interaction

Der source-Relation entspricht die S-Rolle des allgemeinen Rekurssituationstyps, der goalRelation die H-Rolle und der signpost-Relation die P-Rolle.

Dieses allgemeine Ereignis-Modell gründet nach Lutzeier in einem elementaren kognitiven "Batterie-Modell" von Ereignissen: etwas bewegt sich (in der Vorstellung) von einem positiven zu einem negativen Pol, d.h. die Richtung des (vorgestellten) Energieflusses ist ausschlaggebend fur die Einteilung in source (positiver Pol) und goal (negativer Pol). Die 
source-goal-Perspektive ist dann nichts anderes als eine Instantiierung des elementaren Batterie-Modells. Neben der source- und der goal-Relation kann auf dem gerichteten Pfad zwischen source und goal ein drittes Ereigniselement perzipiert bzw. konzipiert werden, das in das gesamte Ereignis involviert ist. Dieses hat die Rolle, zwischen zwei Entitäten einen Pfad zu etablieren. Das Standardbeispiel dafur ist:

(v) Die Lehrerin gibt dem Jungen ein Buch

Das Geben-Ereignis etabliert eine Interaktion zwischen der Lehrerin und dem Jungen: der gerichtete Pfad beginnt bei der Lehrerin und endet bei dem Jungen, d.h. die Lehrerin repräsentiert die source-Relation, dem Jungen die goal-Relation, und das Buch etabliert den Plad zwischen den beiden; die entsprechende NP repräsentiert die signpost-Relation.

Innerhalb dieses Modells ließe sich der Unterschied von anlügen und belügen folgendermaßen erklären: der gerichtete Pfad von source zu goal endet im Fall von anlügen irgendwo auf der Strecke des letzen Drittels, im Fall von belügen endet er auf dem goal-Punkt, im Bild:

Figur 5.

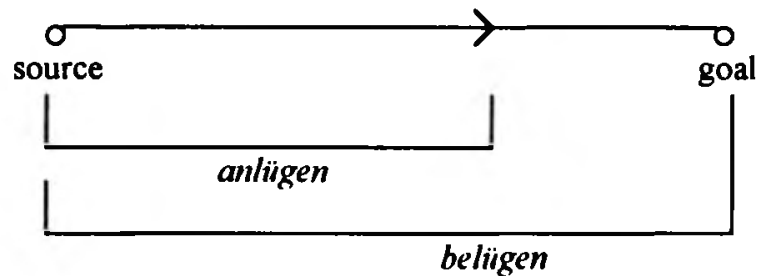

Dies würde zusätzlich noch zweierlei erklären:

(1) Aus dem Satz Er hat ihn belogen folgt Er hat ihn angelogen, aber nicht umgekehrt. Der Satz Er hat ihn stundenlang angelogen ist völlig in Ordnung, wogegen der Satz ?Er hat ihn stundenlang belogen leicht korrupt erscheint.

(2) Die be-Blockierung fur flunkern könnte in diesem Modell so erklärt werden: flunkern hat in dem Lugen-Paradigma den geringsten Intensitätsgrad der Gewichtung der Lüge, ubertragen auf das Batterie-Modell wurde das bedeuten, daß der Energiefluß von source zu goal so gering ist, daß er goal gar nicht erreichen kann.

Zum Abschluß ist das Verhältnis Situationsrolle - Ereignisrolle und syntaktische Form in der folgenden Übersicht dargestellt:

Figur 6.

\begin{tabular}{|l|l|l|} 
Situationsrolle & Ereignisrolle & Formspezifik \\
\hline S & source & NP(Nom) \\
$\mathrm{H}$ & goal & NP(Akk) \\
& & NP(Dat) \\
P & signpost & NP(Akk)/SE \\
& & \\
\hline
\end{tabular}




\section{Literatur}

Austin, J.L. (1962): How to do things with words. - Oxford: Oxford University Press.

Barwise, J. \& Perry, J.(1986): Situationen und Einstellungen. Grundlagen der Situations-semantik. - Berlin: de Gruyter.

Baumgärtner, K. (1977): Lexikalische systeme möglicher performative. - In: Zeitschrift für germanistische Linguistik 5(3), 257-277.

Baumgärtner, K.(1979): Lexikalische systeme möglicher performative. - Ms. Stuttgart.

Coleman, L. \& Kay, P. (1981): Prototype semantics. The English verb 'lie'. - In: Language 57, 26-44.

Lutzeier, R.P.(1981): Wort und Feld. Wortsemantische Fragestellungen mit besonderer Berücksichtigung des Wortfeldbegriffs. - Tübingen: Niemeyer.

Lutzeier, R.P.(1991): Major Pillars of German Syntax. An Introduction to CRMS-Theory. - Tübingen: Niemeyer.

Mudersbach, K.(1984): Kommunikation über Glaubensinhalte. Grundlagen der epistemischen Linguistik. - Berlin: de Gruyter.

Searle, J.R. \& Vanderveken, C.(1985): Foundations of illocutionary logic. - Cambridge: Cambridge University Press.

Storrer, A.(1991): Verbvalenz. Theoretische und methodische Grundlagen ihrer Beschreibung in Grmmatikographie und Lexikographie. - Tübingen: Niemeyer.

Urmson, J.O.(1974): Einstufen. - In: Grewendorf, G. \& Meggle, G. (Hrsg.): Sprache und Ethik (Frankfurr/M.: Suhrkamp), 140-174.

Vanderveken, D.(1990): Meaning and speech acts. - Cambridge: Cambridge University Press.

Verschueren, J.(1980): On speech act verbs. - Amsterdam: Benjamins.

Verschueren, J.(1985): What people say they do with words. - Norwood: New Jersey.

Wiezbicka, A.(1987): English speech act verbs. A semantic dictionary. - Sidney: Academic Press.

Wright, G.H.v.(1977): Handlung, Norm und Intention. Untersuchungen zur deontischen Logik. - Berlin: de Gruyter. 